\title{
Analysis of Propagation Eigenmode for Stripline based on Planar Circuit Equations and Lateral Equivalent Network
}

\author{
Akira HIROTA Takaharu HIRAOKA Jui-Pang HSU \\ Faculty of Engineering, Kanagawa University Yokohama, Japan
}

\begin{abstract}
Stripline/microstrip line is an important waveguide component for MIC. Theoretical treatment of propagation eigenmode for stripline/microstrip line of infinitesimally thin strip has been established. However, theory for thick strip line is not yet established. In order to treat propagation eigenmode of stripline/ microstrip line with thick strip, rigorous equivalent network in the lateral direction is derived based on mode analysis and planar circuit theory. Derived equivalent network is practically applied to the analysis of propagation eigenmode for stripline of any thickness with success.
\end{abstract}

Key Words - stripline, planar circuit equations, lateral equivalent network, propagation eigenmode, number of considered height mode

\section{INTRODUCTION}

Stripline and microstrip line shown in Fig.1 are important waveguides used for microwave integrated circuit. So far stripline or microstrip line circuits has been practically designed without any serious problem even theory of these waveguides is incomplete. The reason is the incomplete theory has been covered by practical measurement. In order to carry out the exact design of microwave circuit by computer, rigorous calculation method for propagation eigenmode (propagation constant and field distribution) of any stripline thickness are strongly needed. However, exact treatment for stripline and micro-stripline structure are only available for infinitesimally thin strip; i.e., exact analysis for the former is carried out by conformal transformation and reasonable analysis for the latter by spectral domain method.

By the way practical stripline has always thickness, whose theory is still incomplete. In order to treat stripline/ microstrip line having thick strip, mode analysis is applied; parallel-plate planar circuit is introduced and the

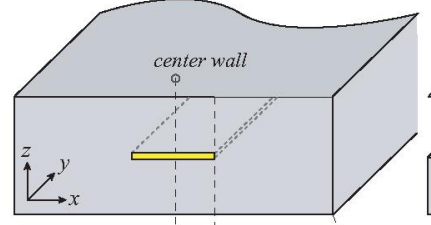

(a) Stripline

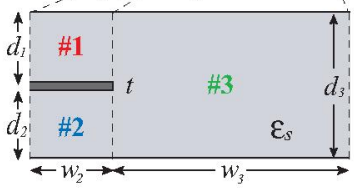

(a') Cross section for stripline

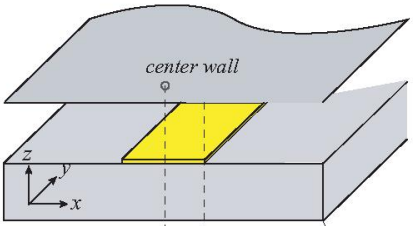

(b) Microstrip line

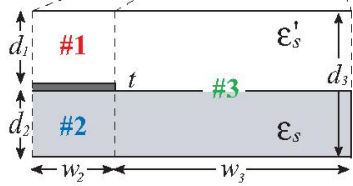

(b') Cross section for MS line

Fig.1 Structure of stripline and microstrip line

equivalent network in the lateral direction is derived. The equivalent network so far developed is applied to the calculation of propagation eigenmode for practical stripline of any strip thickness with success.

\section{Parallel Plate Planar Circutt Equations}

In order to calculate propagation eigenmode of stripline having any thickness shown in Fig.1, half structure of stripline is divided into three regions as shown in Fig.1 $\left(a^{\prime}\right)$. Then each region can be understood as parallel plate planar circuit, whose field can be described by summation of TM mode and TE mode in the parallel plate planar circuit. Field distribution of each TM/TE mode is given by $z$-dependent function and $x-y$ dependent function as shown in Table 1 based on separation of variables and definition of planar voltage and planar current density. $z$-dependent function gives mode function in height direction and $x-y$ dependent function becomes planar circuit equation, where related network parameters are also defined or given in the Table 1.

Table 1

Separation of variables for field distribution of parallel-plate planar circuit $\left(\eta_{0}=\sqrt{\mu / \varepsilon_{0}}[\Omega], k_{0}=\omega \sqrt{\varepsilon_{0} \mu}\right)$

\begin{tabular}{|l|l|l}
\hline (a) separation of variables & (b) $z$-dependent function
\end{tabular}

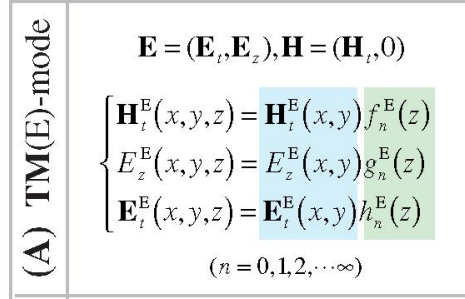

$\mathbf{E}=\left(\mathbf{E}_{t}, 0\right), \mathbf{H}=\left(\mathbf{H}_{t}, \mathbf{H}_{z}\right)$

$\int \mathbf{E}_{t}^{\mathrm{H}}(x, y, z)=\mathbf{E}_{t}^{\mathrm{H}}(x, y) f_{m}^{\mathrm{H}}(z)$

$\left\{H_{z}^{\mathrm{H}}(x, y, z)=H_{z}^{\mathrm{H}}(x, y) g_{n}^{\mathrm{H}}(z)\right.$

$\mathbf{H}_{t}^{\mathrm{H}}(x, y, z)=\mathbf{H}_{t}^{\mathrm{H}}(x, y) h_{m}^{\mathrm{H}}(z)$

$(m=1,2, \cdots \infty)$

$$
\begin{gathered}
f_{n}^{E}(z)=\varepsilon_{s} g_{n}^{E}(z)=\sqrt{\varepsilon_{s}} \sqrt{\varepsilon_{n}} \cos \left(\frac{n \pi}{d} z\right) \\
h_{n}^{E}(z)=-\left(\frac{n \pi}{k_{0} d}\right) \frac{1}{\sqrt{\varepsilon_{s}}} \sqrt{\varepsilon_{n}} \sin \left(\frac{n \pi}{d} z\right) \\
\gamma_{t n}^{E}=\sqrt{\left(\frac{n \pi}{d}\right)^{2}-\varepsilon_{s} k_{0}^{2}}=j \beta_{t n}^{E} \quad \varepsilon_{n}=\left\{\begin{array}{l}
1(n=0) \\
2(n \neq 0)
\end{array}\right. \\
f_{m}^{H}(z)=g_{m}^{H}(z)=\sqrt{2} \sin \left(\frac{m \pi}{d} z\right) \\
h_{m}^{H}(z)=\left(\frac{m \pi}{k_{0} d}\right) \sqrt{2} \cos \left(\frac{m \pi}{d} z\right) \\
\gamma_{t h n}^{H}=\sqrt{\left(\frac{m \pi}{d}\right)^{2}-\varepsilon_{s} k_{0}^{2}}=j \beta_{t m}^{H}
\end{gathered}
$$

(c) $x-y$ dependent function$$
V_{n}^{\bar{E}}(x, y)=-E_{2 x}^{H}(x, y) \cdot d[V] \quad \mathbf{J}_{n}^{E}(x, y)=\mathbf{H}_{s n}^{H}(x, y) \times \mathbf{k}[A / m]
$$$$
\int \operatorname{grad} V_{n}^{E}(x, y)=-j X_{n}^{E} \mathbf{J}_{n}^{E}(x, y)
$$$$
\operatorname{div} \mathbf{J}_{n}^{E}(x, y)=-j B_{n}^{E} V_{n}^{E}(x, y)
$$$$
Y_{c n}^{E}=\sqrt{\frac{B_{n}^{E}}{X_{n}^{E}}}=\frac{\omega \varepsilon_{0}}{\beta_{n}^{E}} \frac{1}{d}[S / m] \quad \beta_{t n}^{E}=\sqrt{X_{n}^{E} B_{n}^{E}}[\mathrm{rad} / \mathrm{m}]
$$$$
V_{m}^{H}(x, y)=H_{z m}^{H}(x, y) \cdot d[A] \quad \mathbf{J}_{m}^{H}(x, y)=\mathbf{k} \times \mathbf{H}_{t m}^{H}(x, y)[V / m]
$$$$
\left\{\operatorname{grad}_{m}^{H}(x, y)=-j X_{m}^{H} \mathbf{J}_{m}^{H}(x, y) \quad X_{m}^{H}=\left(\beta_{m m}^{H}\right)^{2} \cdot d / \omega \mu_{0}[S]\right.
$$$$
\operatorname{div} \mathbf{J}_{m}^{H}(x, y)=-j B_{m}^{H} V_{m}^{H}(x, y) \quad B_{m}^{H}=\omega \mu_{0} / d\left[S / m^{L}\right]
$$$$
Y_{c m}^{H}=\sqrt{\frac{B_{m}^{H}}{X^{H}}}=\frac{\omega \mu_{0}}{\beta^{H}} \frac{1}{d}[\Omega / m] \quad \beta_{m}^{H}=\sqrt{X_{m}^{H} B_{m}^{H}}[\mathrm{rad} / \mathrm{m}]
$$ 


\section{Equivalent Network in Lateral Direction}

In order to calculate propagation eigenmode for stripline structure systematically, equivalent network in lateral direction is derived based on parallel-plate planar circuit equations and dependency of $e^{-j \beta_{\| y}}$ along waveguide. Also half structure of stripline as shown in Fig.2(a) is analyzed because of symmetrical structure.

\section{A. Equivalent network for uniform region}

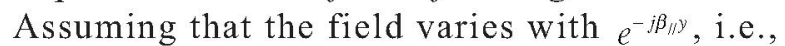
$V\left(x_{y} y\right)=V(x) e^{-j \beta_{j y} y}$ and $J\left(x_{2} y\right)=(J x(x), y(x)) e^{-j \beta_{1, y}}, x$-dependent planar voltage and current density for each mode is given by the following transmission line equations(1),

$$
\left\{\begin{array}{l}
\frac{d V(x)}{d x}=-j X J(x) \\
\frac{d J(x)}{d x}=-j \frac{\beta_{\perp}^{2}}{X} V(x) \quad, \quad \beta_{\perp}^{2}=\beta_{s}^{2}-\beta_{/ /}^{2}
\end{array}\right.
$$

Hence, the equivalent networks for three uniform regions in Fig.2(a) are given by multi-transmission line of TE/ TM as shown in Fig.2(b), whose phase constant and characteristic admittance of parallel( $y)$ and normal $(x)$ direction are given by eqs.(1) and eqs.(2), respectively.

$$
Y_{c / l}=Y_{c} \frac{\beta_{/ I}}{\beta_{t}}, Y_{c \perp}=Y_{c} \frac{\beta_{\perp}}{\beta_{t}}
$$

When $Y_{C I}$ is given by eqs.(2), current density of any mode along waveguide is given by eqs.(3).

$$
J_{y}(x)=Y_{c / /} V(x)
$$

\section{B. Equivalent network for step discontinuity}

Continuity of tangential field at step discontinuity gives following mode coupling equations .

$$
\begin{cases}v^{a \mathrm{H}}=\left(F^{3 H a \mathrm{H}}\right)^{t} v^{3 H} & \vec{J}_{\perp}^{3 H}-i^{3 H}=F^{3 H a \mathrm{H}}\left(\vec{J}_{\perp}^{a \mathbf{H}}-i^{a \mathbf{H}}\right) \\ v^{3 H}=\left(F^{a \mathbf{E} 3 E}\right)^{t} v^{a \mathrm{E}} & \vec{J}_{\perp}^{a \mathbf{E}}-i^{a \mathbf{E}}=F^{a \mathbf{E} 3 E}\left(\vec{J}_{\perp}^{3 E}-i^{3 E}\right)\end{cases}
$$

where mode voltage, mode current in normal direction and converted mode current at both side of step in each region are defined in column matrix with proper suffix meaning port of each region and TE/TM mode; suffix ' $a$ ' means combined mode of region $\# 1$ and $\# 2$ given by the following column matrix.

$$
\mathbf{v}^{a H}=\left(\begin{array}{l}
V^{1 H} \\
V^{2 H}
\end{array}\right), \mathbf{J}^{a H H}=\left(\begin{array}{l}
\mathbf{J}^{1 H} \\
\mathbf{J}^{2 H}
\end{array}\right), i^{a H F}=\left(\begin{array}{l}
i^{1 H} \\
i^{2 H}
\end{array}\right), \mathbf{v}^{a E}=\left(\begin{array}{l}
V^{1 E} \\
V^{2 E}
\end{array}\right), \mathbf{J}^{a E}=\left(\begin{array}{l}
\mathbf{J}^{1 E} \\
\mathbf{J}^{2 E}
\end{array}\right), i^{a E}=\left(\begin{array}{l}
i^{1 E} \\
i^{2 E}
\end{array}\right)
$$

Mode coupling coefficient giving mode coupling matrix are defined in Table 3. Converted mode current column matrix $\boldsymbol{i}$ in eqs.(4) are given by product of mode conversion admittance given in Table 3 and mode voltage as shown in Table 3. Eqs.(4)/ (5) and Tables $2 / 3$ will give equivalent network for step discontinuity, which is given by multi-port ideal transformer and converted mode current source as shown in Fig.2(b). Therefore, whole equivalent network for half structure in lateral direction is given by Fig.2(b), or Fig.2(c) in vector representation. Equivalent network given by vector representation is convenient for matrix operation of analysis which will give propagation eigenmode. Through the derivation of equivalent network in the lateral direction, following relations are used, which are easily proved.

$$
\begin{array}{ll}
H^{3 H 1 E}=F^{3 H 1 H} H^{1 H 1 E} & H^{3 H 2 E}=F^{3 H 2 H} H^{2 H 2 E} \\
H^{1 E 3 H}=F^{1 E 3 E} H^{3 E 2 E} & H^{2 E 3 H}=F^{2 E 3 E} H^{3 H 3 H}
\end{array}
$$

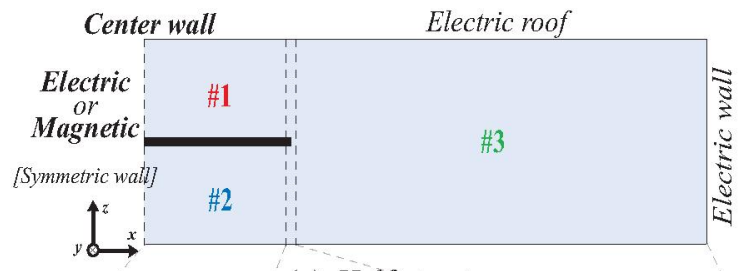

(a) Half structure

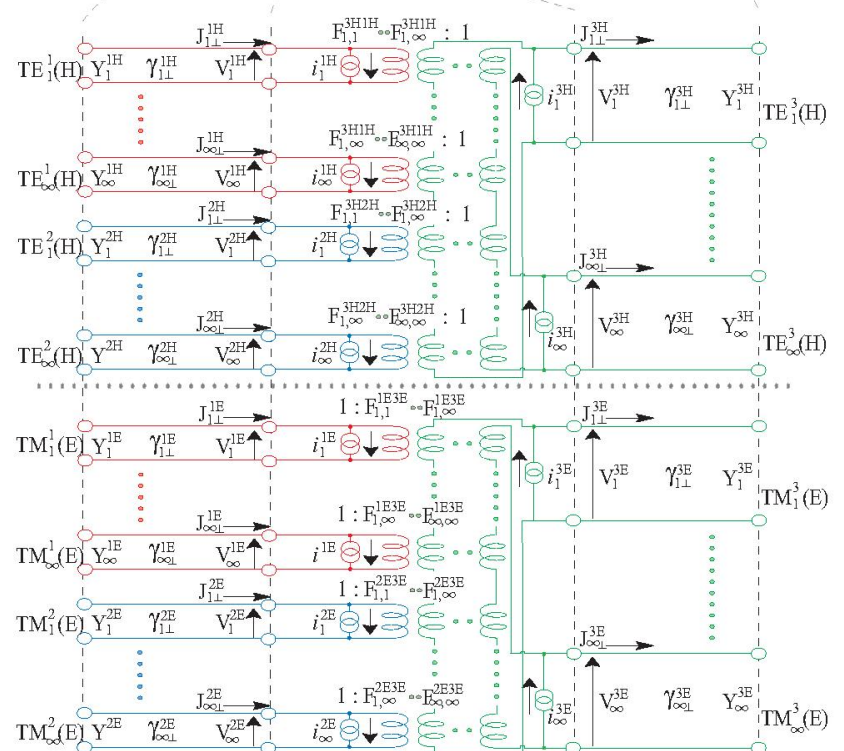

\begin{tabular}{|c|c|}
\hline $\begin{array}{l}Y^{a \mathrm{H} a \mathrm{E}}=j \eta_{0} H^{a \mathrm{H} a \mathrm{E}} Y_{c / /}^{a \mathrm{E}} \\
Y^{a \mathrm{E} a \mathrm{H}}=\frac{1}{j \eta_{0}} H^{a \mathrm{E} a \mathrm{H}} Y_{c / /}^{a \mathrm{H}}\end{array}$ & $\begin{array}{l}{ }^{H 3 E}=j \eta_{0} H^{3 H 3 E} Y_{c / /}^{3 E} \\
{ }^{B E 3 H}=\frac{1}{j \eta_{0}} H^{a \mathrm{E} a \mathrm{H}} Y_{c / /}^{3 H}\end{array}$ \\
\hline $\begin{array}{l}i^{a \mathbf{H}}=Y^{a \mathbf{H} a \mathrm{E}} \mathcal{v}^{a \mathrm{E}}, i^{3 H}=Y^{3 H 3 E} v^{E} \\
i^{3 \mathrm{E}}=Y^{a \mathbf{E} a \mathrm{H}} \mathcal{v}^{a \mathrm{H}}, i^{3 E}=Y^{3 E 3 H} v^{H}\end{array}$ & $\begin{array}{l}J_{/ /}^{a \mathrm{H}}=Y_{c / /}^{a \mathbf{H}} v^{a \mathbf{H}}, J_{/ /}^{3 H}=Y_{c / /}^{3 H} v^{3 H} \\
J_{/ /}^{a \mathrm{E}}=Y_{c / /}^{a \mathrm{E}} v^{a \mathrm{E}}, J_{/ /}^{3 E}=Y_{c / /}^{3 E} v^{3 E}\end{array}$ \\
\hline
\end{tabular}

(b) Equivalent network

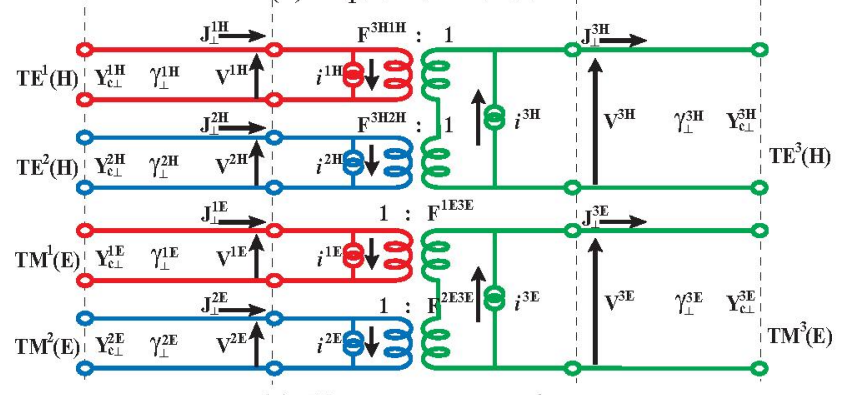

(c) Vector representation

Fig.2 Lateral equivalent network for waveguide

Table 2

TE/TM mode converted admittance

Table 3

Mode coupling and conversion coefficient

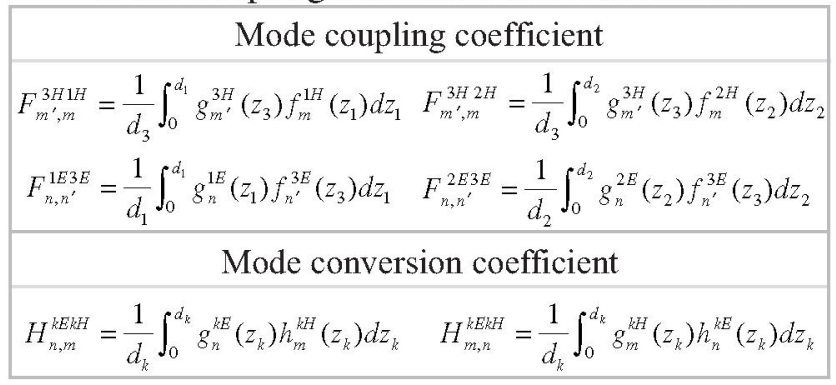




\section{Formulation of Eigenvalue Equation}

Mode matching technique is introduced for formulation of eigenmode equation. TE mode voltage at the step of region \#3 and TM mode voltage at the step of region $\# 1$ and \#2 are assumed to be independent, then TE mode current toward leftward and TM mode current flowing toward rightward are given by the following matrix equation based on the equivalent network and eqs.(4).

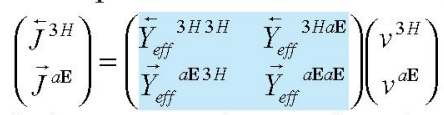

where each admittance matrix are given by eqs.(8)

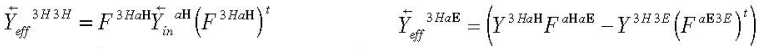

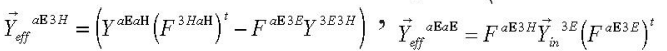

Also TE mode current flowing toward rightward and TM mode current toward leftward are given by the following matrix equation.

$$
\left(\begin{array}{l}
\vec{J}^{3 H} \\
\tilde{J}^{a \mathrm{~B}}
\end{array}\right)=\left(\begin{array}{cc}
\vec{Y}_{i n}^{3 H} & 0 \\
0 & \bar{Y}_{\mathrm{in}}{ }^{a \mathrm{~B}}
\end{array}\right)\left(\begin{array}{c}
v^{3 H} \\
v^{a \mathrm{E}}
\end{array}\right)
$$

where $\boldsymbol{Y}_{\text {in }}$ in eqs.(8) and (9) are input mode admittance and given by Table 4 depending on the outside boundary condition. If there is no wall, $Y_{i n}=Y_{c \mid}$ because of no reflection. Hence, following eigenvalue equation is obtained from mode matching condition.

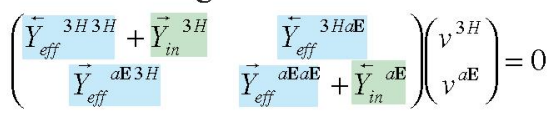

The eigenvalue $\beta_{\|}$gives effective refractive index of each mode and eigen vector can give field distribution of the corresponding mode by using the result of Table 5 .

\section{Calculation of Eigenmode}

Propagation eigenmode of stripline shown in Fig.1(a) is calculated by eqs.(10) and equivalent network shown in Fig.2, whose dimension and parameters are given in the following. Also electric wall is assumed at outside.

$\left\{w_{2}=1.05[\mathrm{~mm}], w_{3}=3.0[\mathrm{~mm}], d_{1}=d_{2}=1.45[\mathrm{~mm}]\right.$,

$$
\left.d_{3}=3.0[\mathrm{~mm}], \quad t=0.1[\mathrm{~mm}], \quad \varepsilon=2.62\right\}
$$

Table 4

\begin{tabular}{|c|c|}
\hline $\mathrm{z}$ & $\begin{array}{l}E_{z}^{k}(x, y, z)=-\frac{1}{d_{k}} \sum_{n} V_{n}^{k E}(x, y) g_{n}^{k E}(z) \\
H_{z}^{k}(x, y, z)=-\frac{1}{d_{k}} \sum_{m} V_{m}^{k H}(x, y) g_{m}^{k i}(z)\end{array}$ \\
\hline$y$ & $\begin{array}{l}H_{y}^{k}(x, y, z)=\sum_{n} J_{n x}^{k E}(x, y) f_{n}^{k E}(z)+j \sqrt{\frac{\varepsilon}{\mu}} \sum_{m} J_{m j}^{k F}(x, y) h_{m}^{k H F}(z) \\
E_{y}^{k}(x, y, z)=-\sum_{m} J_{m a}^{k F}(x, y) f_{m}^{k H}(z)+j \sqrt{\frac{\mu}{\varepsilon}} \sum_{n} J_{n y}^{k E}(x, y) h_{n}^{k E}(z)\end{array}$ \\
\hline $\mathrm{x}$ & 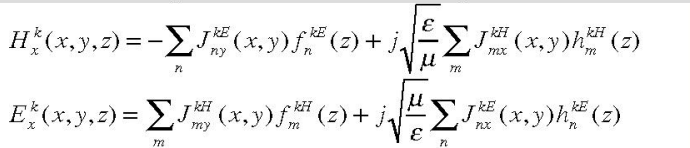 \\
\hline
\end{tabular}

Input admittance depending on side/center wall condition

\begin{tabular}{c|c|c|}
\cline { 2 - 3 } & Electric Wall & Magnetic Wall \\
\hline TM(E)-mode & $\boldsymbol{Y}_{i n}^{E}=\boldsymbol{Y}_{c \perp}^{E} \operatorname{coth}\left(\gamma_{\perp}^{E} W\right)$ & $\boldsymbol{Y}_{i s}^{E}=\boldsymbol{Y}_{c \perp}^{E} \tanh \left(\gamma_{\perp}^{E} W\right)$ \\
\hline TE(H)-mode & $\boldsymbol{Y}_{i n}^{H}=\boldsymbol{Y}_{c \perp}^{H} \tanh \left(\gamma_{\perp}^{H} W\right)$ & $\boldsymbol{Y}_{i n}^{H}=\boldsymbol{Y}_{c \perp}^{H} \operatorname{coth}\left(\gamma_{\perp}^{H} W\right)$ \\
\hline
\end{tabular}

Table 5

Field description in $k$-th region by mode summation
In our calculation thickness of stripline and operational frequency are widely varied including zero thickness. Key point of our method is number of height mode to be considered, which is decided by convergence behavior of propagation constant with number of height mode in region \#3. These calculated results are shown in Fig.5(a) for second and third mode with $0.1[\mathrm{~mm}]$ thickness. From these results $50 \mathrm{TE} / \mathrm{TM}$ height modes are taken into consideration for calculation of propagation constant.

\section{A. Calculation of dispersion curves}

Propagation constant vs frequency for $0.1[\mathrm{~mm}]$ thickness stripline are calculated and shown in Fig.3, which agree well with that for no-thickness case calculated by the same theory. Calculated propagation constant for dominant mode agree with TEM mode propagation con$\operatorname{stant}\left(\beta_{t 0}=\beta_{/ /}=k_{0} \sqrt{\varepsilon_{0}}\right)$.

\section{B. Field distribution of propagation eigenmode}

Transverse field distribution of lower three eigenmodes in the waveguide cross-section for $0.1[\mathrm{~mm}]$ thickness stripline at $60[\mathrm{GHz}]$ is shown in Fig.4, where 1st mode is TEM and 2 nd $/ 3$ rd mode are TM modes. Electric field distribution $E z$ of dominant TEM mode are calculated and shown in Fig. 6 for various stripline thickness including no thickness. No thickness case is similar with $t=0.1[\mathrm{~mm}]$ case shown in Fig.4(a) but is not agreed exactly. Number of considered TE/TM height mode is 100 . C. Convergence of field with number of height mode

Convergence behavior of field distribution with number of height mode for TEM dominant mode is investigated and that of $E z$ component (field in height direction) are shown in Fig5 $(b)$, where number of TE/TM height mode taken into consideration is 20,50 and 100 . From these results we can see that the field distribution surely converges with number of mode, but convergence is slow because of sharp edge at the corner.

\section{Conclusion}

Rigorous lateral equivalent network for stripline is derived based on parallel plate planar circuit equations and mode analysis. The derived equivalent network is practically applied to the calculation of eigenmode for stripline structure with success. Advantage of this method are (1) method is systematic and appropriate for computer, (2) physical situation in waveguide becomes clear by mode analysis and (3) main calculation error is caused by truncation of mode, which will give the reasonable estimation of error vs CPU time relation. Thus derived equivalent network will be useful for analysis of any other waveguide such as micro-stripline and slotted line.

\section{REFERENCES}

[1]Hsu,Jui-Pang and T.Anada,"Proposal of Surface-Wave Planar Circuit, Formulation of its Planar Circuit equations and its Practical Application",1986 IEEE MTT-S Digest., GG-4,pp797-800 [2]Hsu,Jui-Pang and T.Anada ,"Formulation of mode coupling equations at step discontinuity based on the planar circuit theory",1989 IEEE MTT-S Digest.,PP-5, pp1135-1138

[3]S.T.Peng and A.A.Oliner,"Guidance and leakage properties of a class of open dielectric waveguides:Part I,Part II",IEEE Trans. MTT, Vol.MTT29,pp843-855, Sep.1981. 


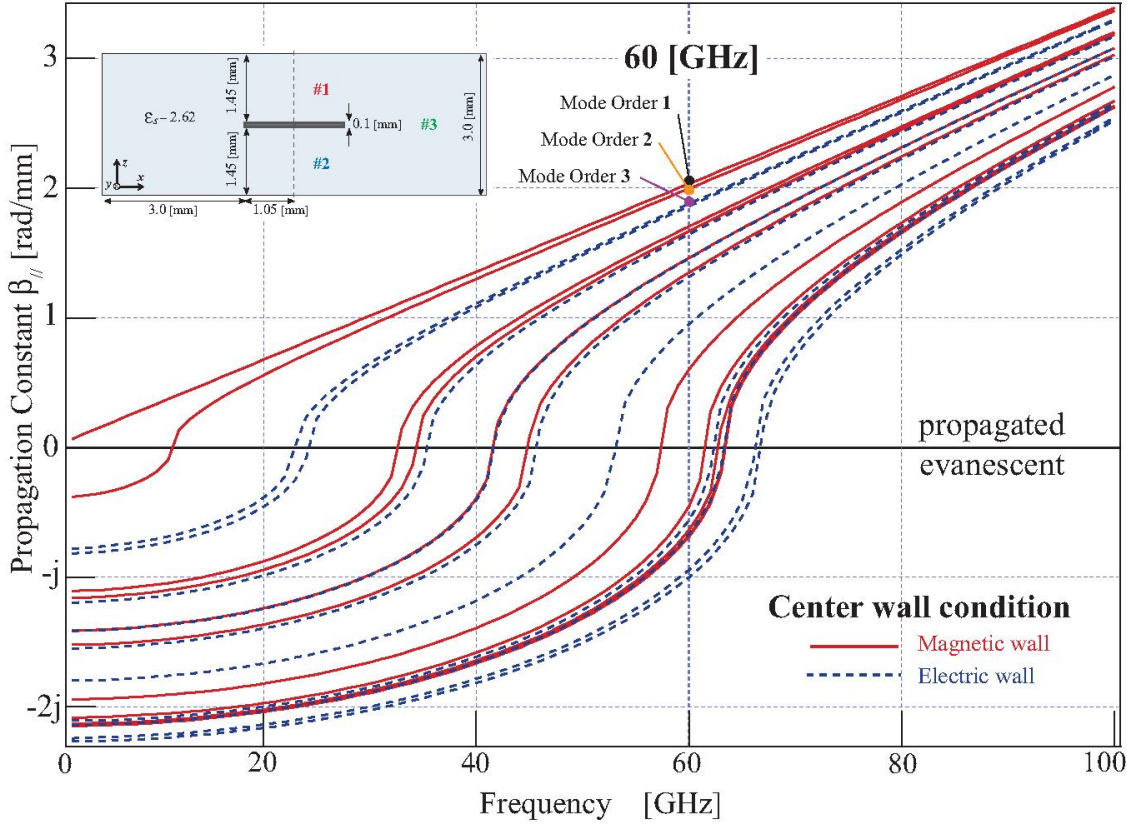

Fig.3 Dispersion curves and dimension for $0.1[\mathrm{~mm}]$ thickness stripline -- considered mode : 100 --

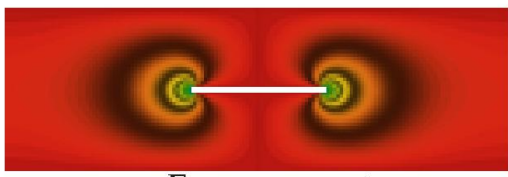

Ex component

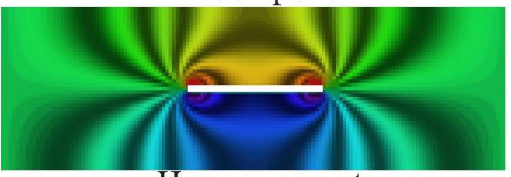

Hx component

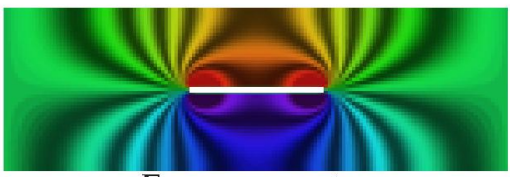

Ez component

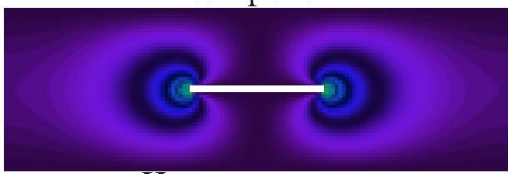

$\mathrm{Hz}$ component (a) Mode order 1 (TEM-mode) (Magnetic wall at the center)

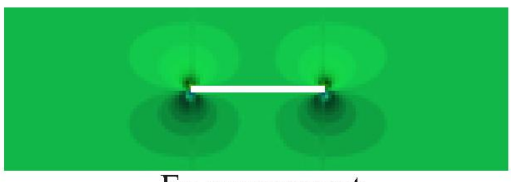

Ex component

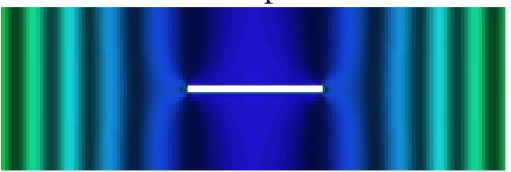

Hx component

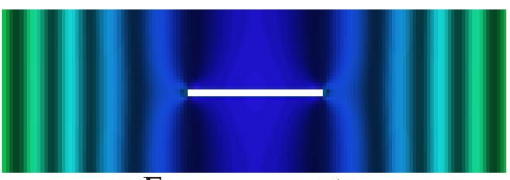

Ez component

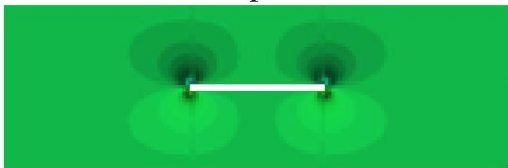

Hz component (b) Mode order 2 (TE-mode) (Magnetic wall at the center)

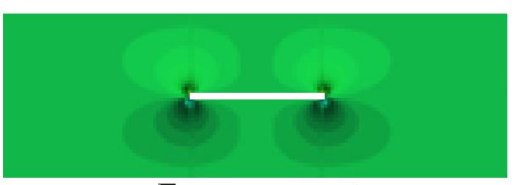

Ex component

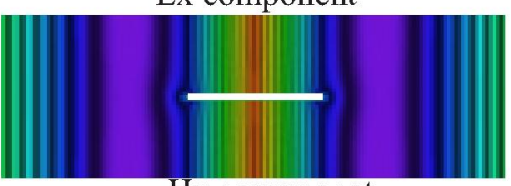

Hx component

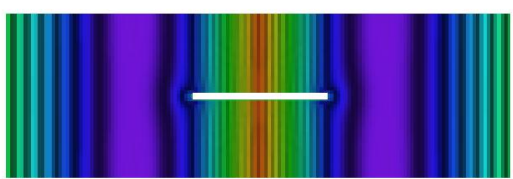

Ez component

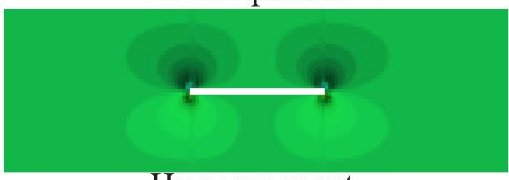

Hz component (c) Mode order 3 (TE-mode) (Electric wall at the center)

Fig.4 Field distribution of lower three eigenmode at $60[\mathrm{GHz}]$ -- considered mode : $100, t=0.1[\mathrm{~mm}]$--

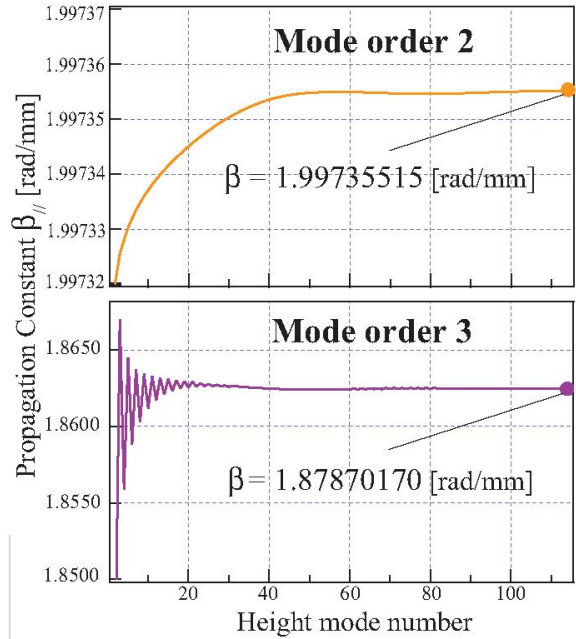

(a) Convergence of propagation constant with height mode number

\section{Ez component}

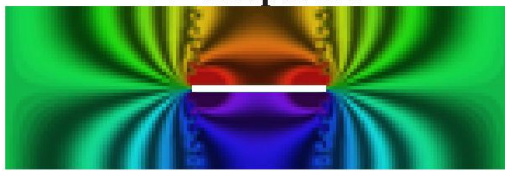

(b-l) $\mathbf{2 0}$ modes considered

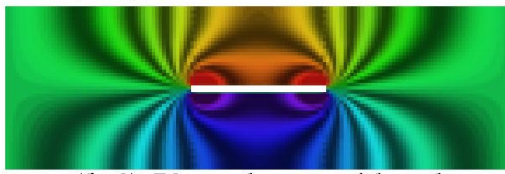

(b-2) $\mathbf{5 0}$ modes considered

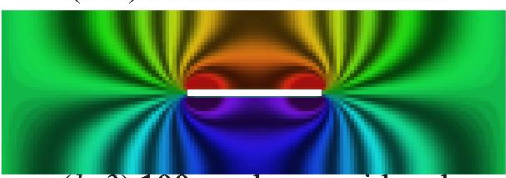

(b-3) $\mathbf{1 0 0}$ modes considered

(b) Convergence of $E z$ field component with height mode number

Fig.5 Convergence of eigenmode with height mode number $--t=0.1[\mathrm{~mm}]--$

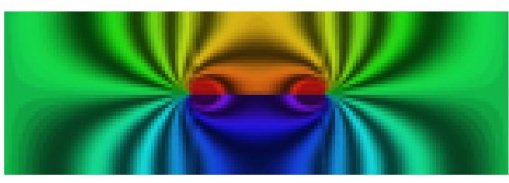

(a) Ez component , $\mathbf{t}=\mathbf{0 . 0}[\mathrm{mm}]$

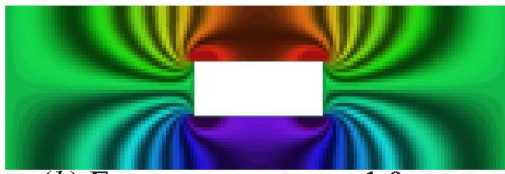

(b) Ez component, $\mathrm{t}=\mathbf{1 . 0}[\mathrm{mm}]$

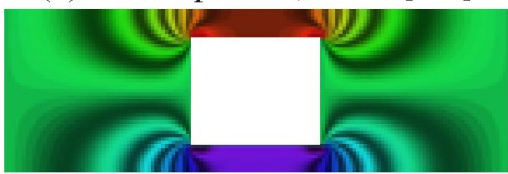

(c) Ez component, $\mathrm{t}=\mathbf{2 . 0}[\mathrm{mm}]$

Fig.6 Field distribution of eigenmode for various stripline thickness at $60[\mathrm{GHz}]$ ---- considered mode : 100 -- 